\title{
Formation of Composite Surface during Friction Surfacing of Steel with Aluminium
}

\author{
S. Janakiraman and K. Udaya Bhat \\ Department of Metallurgical \& Materials Engineering, NITK Surathkal, Srinivasa Nagar, Surathkal 575025, India \\ Correspondence should be addressed to K. Udaya Bhat, udayabhatk@gmail.com
}

Received 18 May 2012; Revised 15 August 2012; Accepted 29 August 2012

Academic Editor: Patrick De Baets

Copyright ( $) 2012$ S. Janakiraman and K. U. Bhat. This is an open access article distributed under the Creative Commons Attribution License, which permits unrestricted use, distribution, and reproduction in any medium, provided the original work is properly cited.

Commercial pure aluminium was deposited on medium carbon steel using friction surfacing route. An aluminium rod was used as the consumable tool. Normal load and tool rotation speed were the variables. Under certain combinations of load and speed the deposition was continuous and uniform. The deposit consisted of $\mathrm{Al}$ embedded with fine particles of iron. The interface between substrate material and deposited material was smooth and relatively small. A mechanism is discussed for formation of a composite surface on the steel substrate.

\section{Introduction}

Steel remains one of the important structural material because of its relatively low cost, high processability, manipulation of the properties using principles of alloy design, heat treatment, and so forth [1]. Unfortunately, it's service properties like corrosion resistance, oxidation resistance, are not very good. This limitation arises because the oxide layer forming on the surface of the steel is a noncompact one [1]. This limitation can be overcome by modifying the surface of the steel appropriately, either by changing the surface chemistry (alloying at the surface) or by deposition of another metal at the surface [2]. The deposited metal may on its own give beneficial properties or after appropriate conversion. On this count, deposition of a thin layer of aluminium on steel is very relevant. Aluminium layer, when it is oxidized, forms a compact oxide layer, protecting the substrate steel from oxidation, corrosion, and abrasion [3, 4]. A thin layer of $\mathrm{Al}$ on steel can be obtained by various means, that is, liquid route and solid route. Hot dip aluminising is a predominant method using liquid route, but this route is handicapped by the formation of brittle intermetallics [5]. Chemical routes like pack aluminising do not involve use of liquid state, but they also involve processing at elevated temperatures for long duration, again giving rise to intermetallics and grain growth in the substrate. In this context friction surfacing is a promising route. It can produce an aluminium layer on the steel substrate [6], and if a compact oxide layer is required top layer can be made to undergo oxidation. In friction surfacing the surface of a component is modified using mechanical energy generated using a friction tool [7]. An alloying element can be added during friction surfacing which will be mixed with the substrate to generate an alloyed surface. In friction surfacing, the tool is a consumable one and depending on the relative strengths of substrate and tool materials, as well as temperature attained, both substrate and tool or only tool material will be undergoing plastic deformation. This will lead to alloying near the surface leading to a change in the surface properties [8]. In friction surfacing of steel with aluminium, steel is used as substrate and aluminium is the consumable tool. If friction surfacing parameters are appropriate it is possible to obtain a uniform aluminium deposition on the steel surface [6]. Aluminium layer will have steel (iron) particles embedded in it producing an iron-aluminum composite layer on the steel surface [6]. This paper discusses formation of such composite layer during friction surfacing (of steel with aluminium).

\section{Materials and Experimental Methods}

2.1. Materials and Processing. Medium carbon steel plate $(\mathrm{C}=$ $0.35, \mathrm{Mn}=0.65, \mathrm{P}=0.03, \mathrm{~S}=0.04$; all are in $\mathrm{wt} . \%)$ was 
TABLE 1: Processing parameters for various samples and quality of the deposition.

\begin{tabular}{lcccl}
\hline Trial no. & Tool travel speed $(\mathrm{mm} / \mathrm{min})$ & Load $(\mathrm{kN})$ & Spindle speed $(\mathrm{rpm})$ & Quality of the deposition \\
\hline T1 & 35 & 3 & 200 & Powdery deposition, \\
T2 & 35 & 3 & 400 & Deposition better than T1, still powdery \\
T3 & 35 & 4 & 200 & Discontinuous and varying width. \\
T4 & 35 & 4 & 400 & Good (uniform, continuous) deposition \\
T5 & 35 & 5 & 200 & Continuous, varying width \\
T6 & 35 & 5 & 400 & Good deposition, Width more than T4 \\
\hline
\end{tabular}

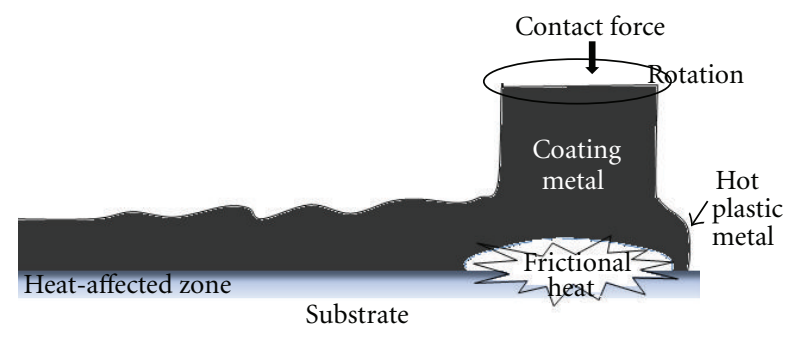

FIGURE 1: Scheme of friction surfacing [8].

taken as the substrate. Substrate dimensions were $150 \mathrm{~mm}$ length, $70 \mathrm{~mm}$ width, and $8 \mathrm{~mm}$ thickness. Controlled roughness on the steel substrate was obtained by milling the substrate using a conventional surface milling machine. Depth of groves produced during milling was measured using cross-sectional microscopy and it was in the range of 25-32 micrometers (um). Roughness of the milled surface was measured using a Veeco optical profile meter. Measured roughness $(\mathrm{Ra})$ was in the range of $5.8 \mathrm{um}$ to $8.3 \mathrm{um}$. Commercial pure $\mathrm{Al}$ ( $99.6 \%$ pure), available in the form of extruded rod, was used as the consumable tool. Extruded rod was machined to a dimension of $100 \mathrm{~mm}$ length and $25 \mathrm{~mm}$ diameter and it was used for deposition. The friction surfacing was done using the machine made by M/s ETA technologies, Bangalore, India. Figure 1 shows a schematic presentation of friction surfacing [8].

$\mathrm{Al}$ was deposited using different processing conditions. Normal load was varied as $3 \mathrm{kN}, 4 \mathrm{kN}$, and $5 \mathrm{kN}$. This gave a stress level of $6.1 \mathrm{MPa}, 8.1 \mathrm{MPa}$, and $10.2 \mathrm{MPa}$ in the consumable tool. Tool spindle speed was varied as $200 \mathrm{rpm}$ and $400 \mathrm{rpm}$. Tool plunge depth was fixed at $40 \mathrm{~mm}$. Tool plunge depth is the total depth up to which the tool can be lowered in the machine. For all experiments tool travel speed was fixed as $35 \mathrm{~mm} / \mathrm{min}$. For convenience the samples were labelled as T1, T2, .., T6 and they are listed in Table 1. All the experiments were done in open atmosphere conditions and for $200 \mathrm{~s}$.

2.2. Characterisation of the Deposit. Quality of the deposition was investigated using various parameters, namely, nature of the deposition (powdery or not), continuity, width uniformity. This information is also listed in Table 1. Morphological investigation, composition of the deposit, and cross-sectional microscopy were done using Scanning Electron Microscope (SEM) with an EDS attachment. Phase identification of the deposit was made using X-ray diffractometry (XRD) using $\mathrm{Cu}_{K \alpha}$ radiation.

\section{Results and Discussion}

3.1. Quality of the Deposition. Quality of the deposition was decided based on the macroobservation using normal eye or low-magnification tools (magnification up to 10x). Deposits were either powdery, patchy (discontinuous and varying width), or continuous. Within the continuous group, width could be uniform or nonuniform. For good coverage of the surface, continuous (preferably with uniform width) deposition is essential. Figure 2 shows macroimage of the sample T6. The track length was about $30 \mathrm{~cm}$ and width was in the range of $25-30 \mathrm{~mm}$. From the macroimage we could conclude that the deposition was continuous and of almost uniform width. Quality of the deposition is closely related to heat input at the interface and partition of heat between the substrate and the tool. The heat input at the interface (HI) could be written as [7]

$$
\text { Heat input }(\mathrm{HI})=\frac{\text { Power input }}{\text { scan speed }} .
$$

Extrapolating the concept from friction stir welding [7] power input (PI) could be written as a function of spindle speed $(s)$ and torque $(M)$ :

$$
\mathrm{PI}=s * M .
$$

For the sake of simplicity, the energy losses associated with the drives and transmission systems were neglected. Mechanical energy available at the substrate-tool interface was partitioned in to heat and deformation component required for local plastic deformation $[10,11]$. The heat input at the interface gets dissipated predominantly by conduction. The temperature rise at the interface was sufficient to make aluminium near the interface plastic, and the relative sliding of the tool with respect to the substrate leads to transfer of the plastic material as a thin layer. This explains why good deposition was seen only under certain combinations of load and rotation speed (other parameters were kept constantin our experiments). It may be mentioned that only aluminium side became plastic, whereas the temperature rise was not sufficient to make steel plastic.

3.2. Topographical Details. Figure 3(a) shows a low-magnification image observed under SEM in secondary electron mode. It may be noted that topographic information is 


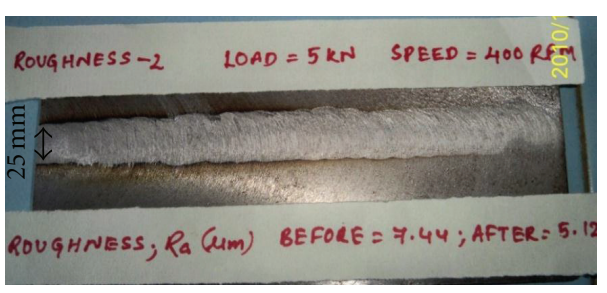

(a)

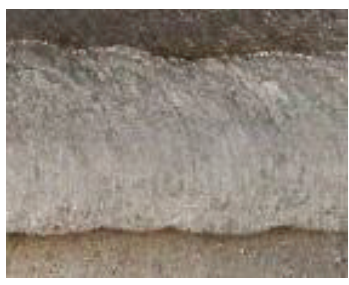

(b)

Figure 2: Macroimage of the deposition made using a normal load of $5 \mathrm{kN}$ and spindle speed of $400 \mathrm{rpm}$. On the right side a small region which was magnified from long track is shown.

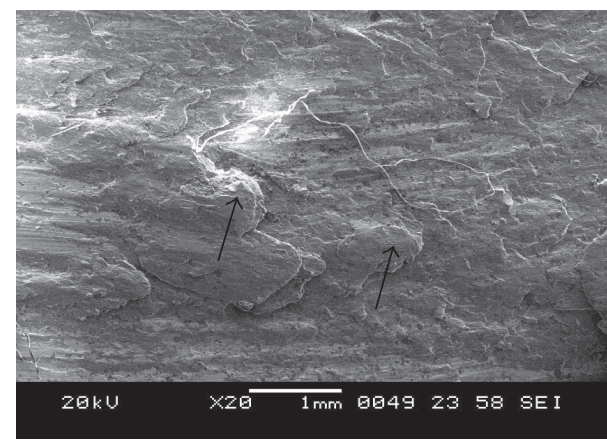

(a)

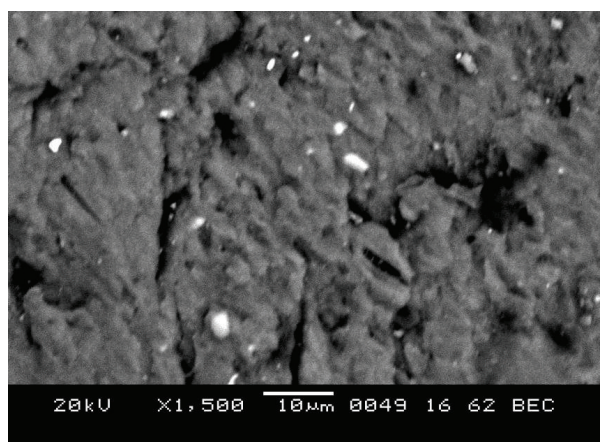

(c)

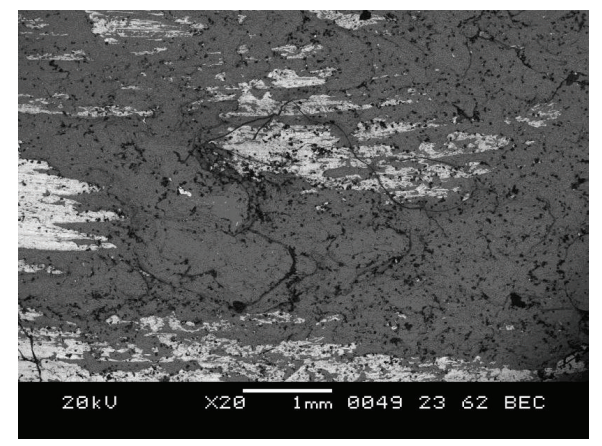

(b)

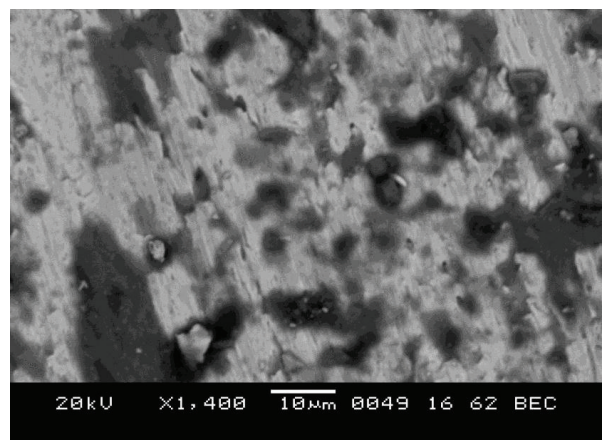

(d)

FIGURE 3: (a) A low-magnification micrograph of friction-surfaced region. (b) SEM: backscattered electron-compositional (BEC) image. (c) and (d) are micrographs taken from Fe-rich and Al-rich regions of Figure (b). In Figure (c) Al-rich region with fine Fe particles is observed. In (d) Fe-rich region has embedded small Al particles.

better revealed in this mode [12]. There is no marking typical of surface milling process. Surface has occasional microhills (indicated by arrow marks) on the flat surface. The backscattered electron-compositional micrograph (BEC image) presented in Figure 3(b) shows two types of contrast, namely, white region which is predominantly iron and grey region which is predominantly aluminium. It must be noted that both micrographs (Figures 3(a) and 3(b)) are taken from same region and under same magnification. By comparing topographic information (Figure 4(a)) and compositional information (Figure 4(b)) we conclude that topographical variations observed in Figure 3(a) is not due to presence of iron-rich and aluminium-rich regions in the deposited layer.

3.3. Cross-Sectional Microscopy. Figure 4(a) shows cross sectional view of the deposited region. Deposition thickness is fairly uniform, and measurement over $1 \mathrm{~mm}$ length gave thickness in the range of 90 to $106 \mathrm{um}$. Interface is macroscopically smooth, without any profiles created during surface milling. This is more clearly visible in Figure 4(b). Figure 4(b) presents interface between substrate materialdeposit material. The interface is relatively smooth and small. The compositional profile across the interface (Figure 4(c)) shows minimum (almost zero) level of mixing of species on either side of the interface. This statement is without considering the material transfer in the form of particles which are visible as white particles (iron) embedded in the deposited aluminium. Figure 4(d) shows a high-magnification micrograph from Figure 4(a). We see iron particles of nanometer scale (arrow pointers in Figures 4(b) and 4(d)) embedded in aluminium matrix. The average spacing between the particles is also very small, indicating that they would contribute 


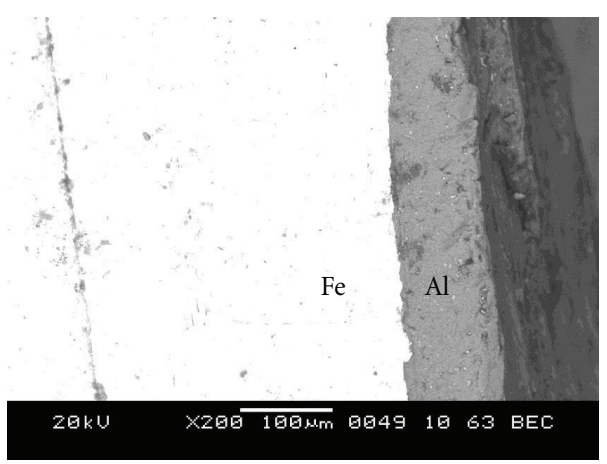

(a)

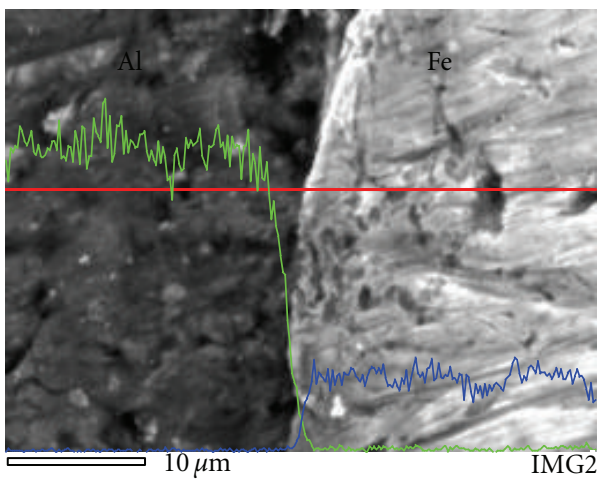

(c)

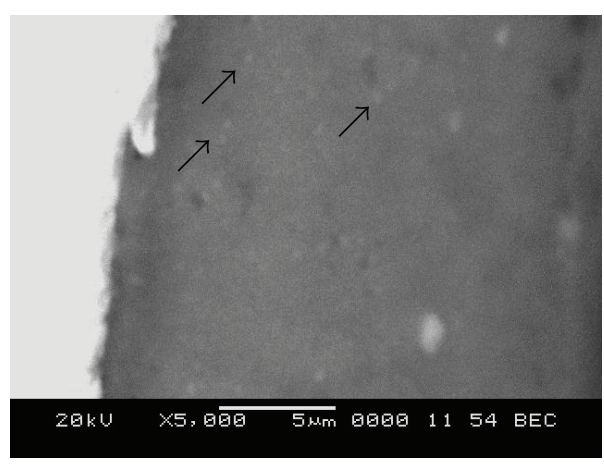

(b)

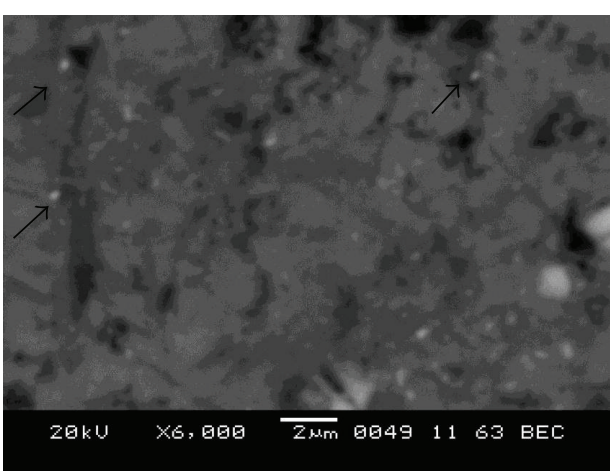

(d)

FIgURE 4: (a) Cross-sectional microscopy showing a uniform deposition. (b) Nature of Fe-Al deposit interface. (c) Smooth and thin interface between aluminium and iron. (d) Fine Fe particles embedded in deposited Al.

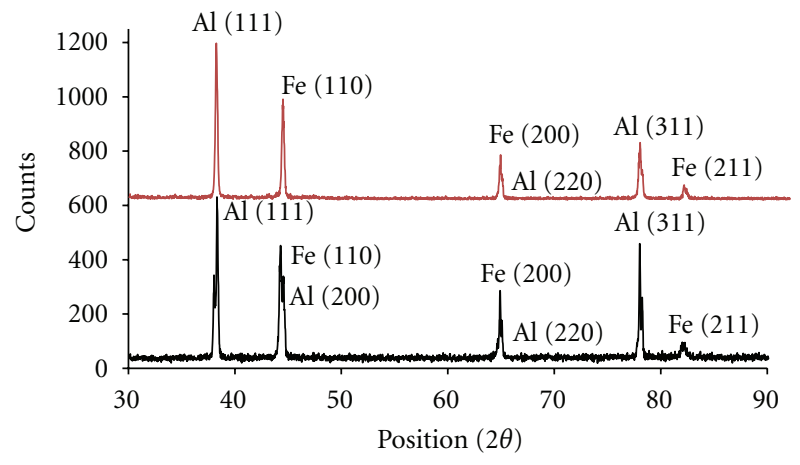

Figure 5: XRD plots of two samples, namely, T5 (top) and T6 (bottom).

for particle strengthening [13]. Strong Fe particles are expected to strengthen soft $\mathrm{Al}$ matrix.

3.4. Phase Identification. Figure 5 shows the XRD analysis of two samples (viz. T5 and T6). XRD plots for other samples are similar. XRD plot indicates that deposit consists of iron and aluminium. From microstructural observations and XRD results we say that the deposit is a mechanical mixture of aluminium and iron. There are no other phases (Fe-Al intermetallics) formed which could be detected by the XRD.
3.5. Formation of Composite Layer during Friction Surfacing. From XRD and SEM study we can conclude that the deposit is a mechanical mixture of aluminium and iron. Since, the consumable tool was pure $\mathrm{Al}$, during friction surfacing, iron particles must have formed and got mixed with aluminium. This has resulted in the deposition of a composite layer of aluminium and steel. Formation of a composite layer is similar to material transfer during friction conditions [9] and can be explained as follows.

In the beginning of friction surfacing both surfaces have asperities. These asperities have various dimensional scales. This means that only few asperities are in contact with each other forming a contact pair [14]. The effective stress at the contact point may be very high compared to the average stress estimated using normal load and initial section diameter. When there is a relative sliding between two surfaces, the asperities will undergo deformation. Being a weaker material, the plastic deformation will be much more towards $\mathrm{Al}$ side than Fe side. Actual strain value will be very high, and it will vary depending on the morphologies of the asperities. Al, though more ductile, may get fractured easily, because of poor strength value. But being fresh surfaces, two Al surfaces have a chance to get rewelded. On the other hand, Fe is a strain hardenable material and at the asperity contact they will become hard, brittle, and get sheared during sliding. Even though fracture surface is clean and fresh, owing to smaller $T / T_{m}$ ( $T$ is the interface temperature, 
Shearing direction
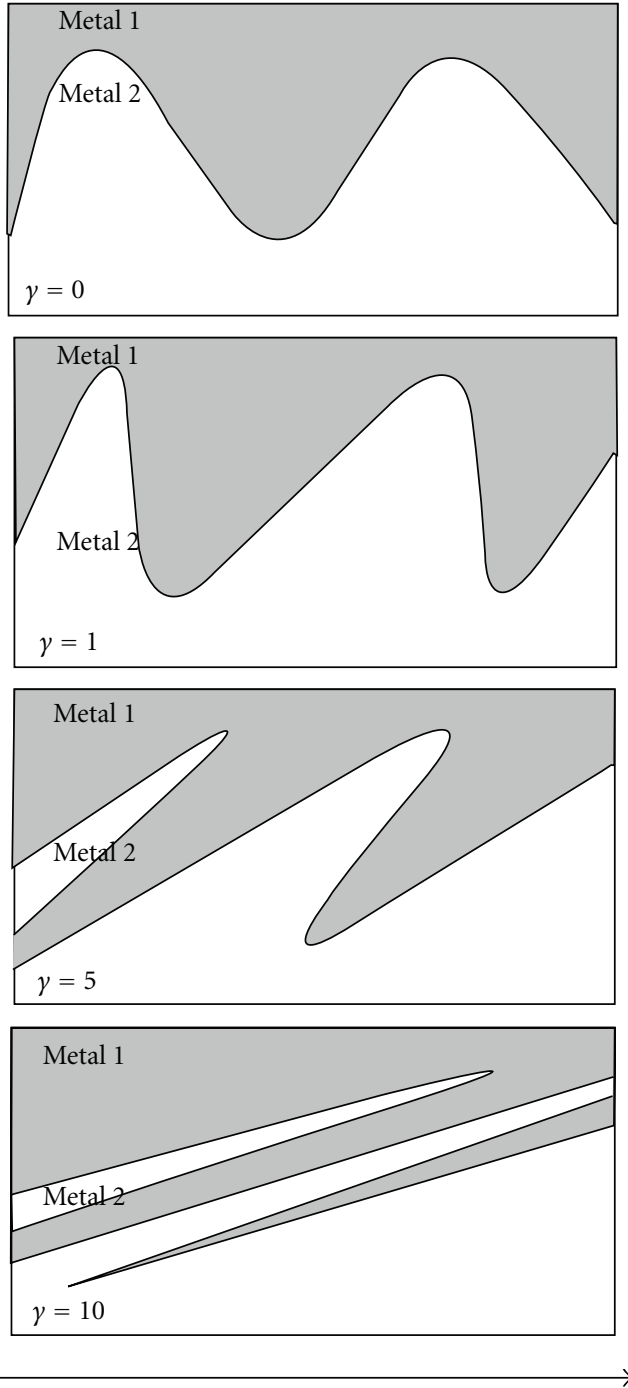

FIGURE 6: Scheme leading to asperity tip fracturing and incorporation into second material during sliding under friction conditions [9]. $\gamma$ : shear strain.

$T_{m}$ is the melting point, both are in kelvins) for steel, the chances of them to get rewelded are small. Figure 6 shows schematic methodology in fracturing of asperity tip at the friction contact. The Fe-Al interface shown in Figure 4(b) shows reduced grove depth (less than $5 \mathrm{um}$ ) compared to initial grove depth (25-32 um). This supports the argument that the asperity hills on hard Fe surface get broken during shearing. Broken Fe particles get mixed up in soft Al layer and the mixture gets deposited during friction surfacing.

\section{Conclusions}

Based on the experimental results, the following conclusions are drawn. A thin layer of $\mathrm{Al}$ can be deposited on steel surface using friction surfacing method. Deposited Al consisted of small Fe particles dispersed in it. Deposit is a mechanical mixture of $\mathrm{Al}$ and $\mathrm{Fe}$. The interface between substrate material and deposited material is smooth and relatively sharp. A mechanism for the formation of a composite layer is presented using shearing, mixing, and deposition of plastic material during surfacing.

\section{Acknowledgment}

The authors thank the Director of National Institute of Technology Karnataka, Surathkal, India, for the permission, financial assistance, and appreciation, extended to carry out this investigation.

\section{References}

[1] W. T. Lankford, N. L. Samways, R. F. Craven, and H. E. McGannor, The Making, Shaping and Treating of Steel, USS, 10th edition, 1985.

[2] K. G. Budhinski and M. K. Budinski, Engineering Materials, Properties and Selection, PHI Learning Pvt Ltd., New Delhi, India, 9th edition, 2009.

[3] A. Nishimoto and K. Akamatsu, "Microstructure and oxidation resistance of Fe3A1 coatings on austenitic stainless steel by spark plasma sintering," Plasma Processes and Polymers, vol. 6, no. 1, pp. S941-S943, 2009.

[4] Z. Xiao-Lin, Y. Zheng-Jun, G. Xue-Dong, C. Wui, and Z. Ping-Ze, "Microstructure and corrosion resistance of Fe$\mathrm{Al}$ intermetallic coating on 45 steel synthesises by double glow plasma surface alloying technology," Transactions of Nonferrous Metals Society of China, vol. 19, pp. 143-148, 2009.

[5] G. Eggeler, W. Auer, and H. Kaesche, "Reactions between low alloyed steel and initially pure as well as iron-saturated aluminium melts between 670 and 800 degree c," Zeitschrift fuer Metallkunde, vol. 77, no. 4, pp. 239-244, 1986.

[6] S. Janakiraman, J. Reddy, S. V. Kailas, and K. Udaya Bhat, "Surface modification of steels using friction stir surfacing," Materials Science Forum, vol. 710, pp. 258-263, 2012.

[7] R. S. Mishra, M. W. Mahoney, S. X. McFadden, N. A. Mara, and A. K. Mukherjee, "High strain rate superplasticity in a friction stir processed $7075 \mathrm{Al}$ alloy," Scripta Materialia, vol. 42, no. 2, pp. 163-168, 1999.

[8] G. W. Stachowiak and A. W. Batchelor, Engineering Tribology, Elsevier, Singapore, 3rd edition, 2005.

[9] J. L. Young Jr., D. Kuhlmann-Wilsdorf, and R. Hull, "The generation of mechanically mixed layers (MMLs) during sliding contact and the effects of lubricant thereon," Wear, vol. 246, no. 1-2, pp. 74-90, 2000.

[10] S. Cui, Z. W. Chen, and J. D. Robson, "A model relating tool torque and its associated power and specific energy to rotation and forward speeds during friction stir welding/processing," International Journal of Machine Tools and Manufacture, vol. 50, no. 12, pp. 1023-1030, 2010.

[11] Y. J. Chao, X. Qi, and W. Tang, "Heat transfer in friction stir welding: experimental and numerical studies," ASME Journal of Manufacturing Science and Engineering, vol. 125, pp. 138145, 2003.

[12] P. J. Goodhew, J. Humphreys, and R. Beanland, Electron Microscopy and Analysis, Taylor and Francis, London, UK, 3rd edition, 2001.

[13] G. E. Dieter, Mechanical Metallurgy, McGraw Hill, London, UK, 1988.

[14] H. Zhai and H. Zhang, "Instabilities of sliding friction governed by asperity interference mechanisms," Wear, vol. 257, pp. 414-419, 2004. 

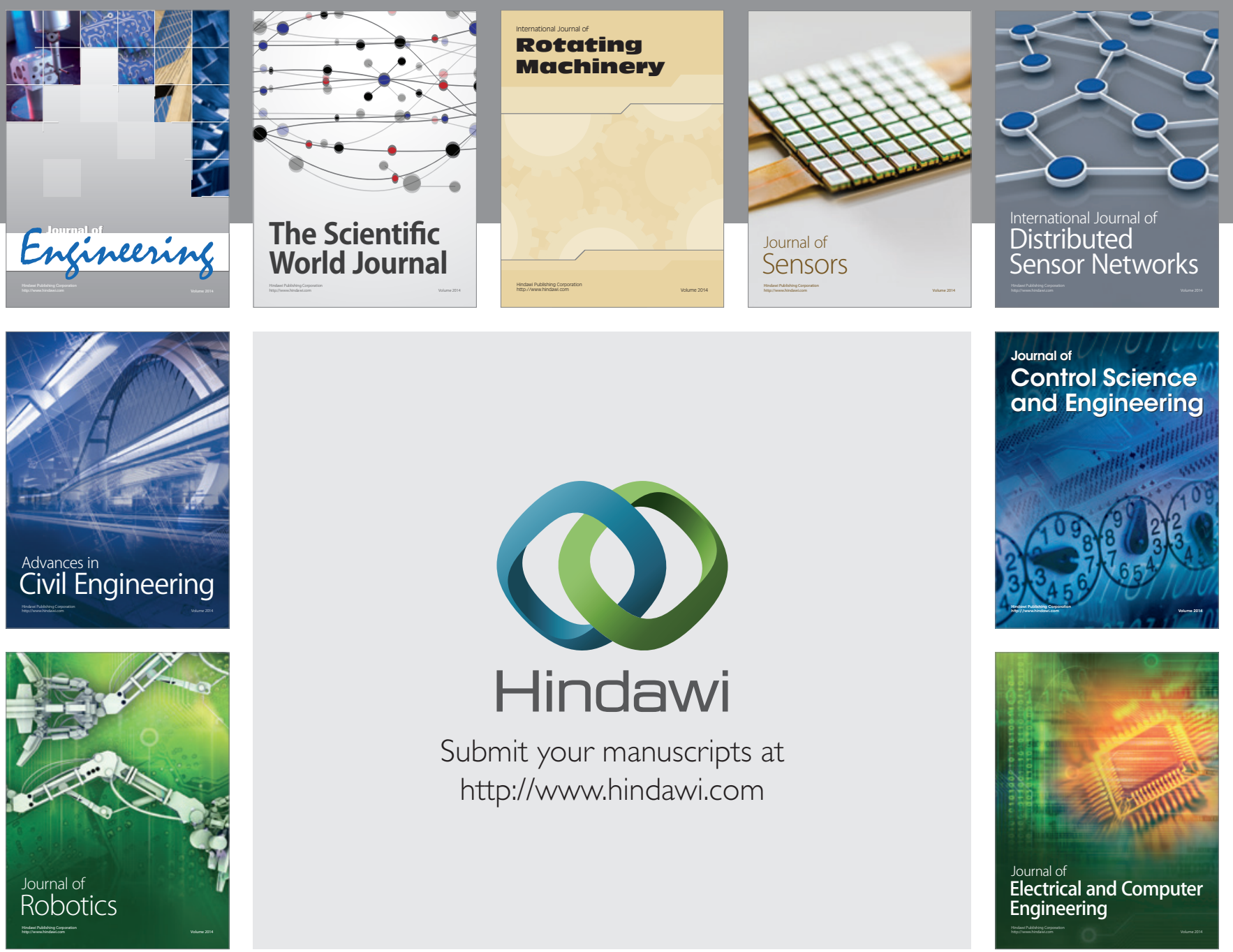

Submit your manuscripts at

http://www.hindawi.com
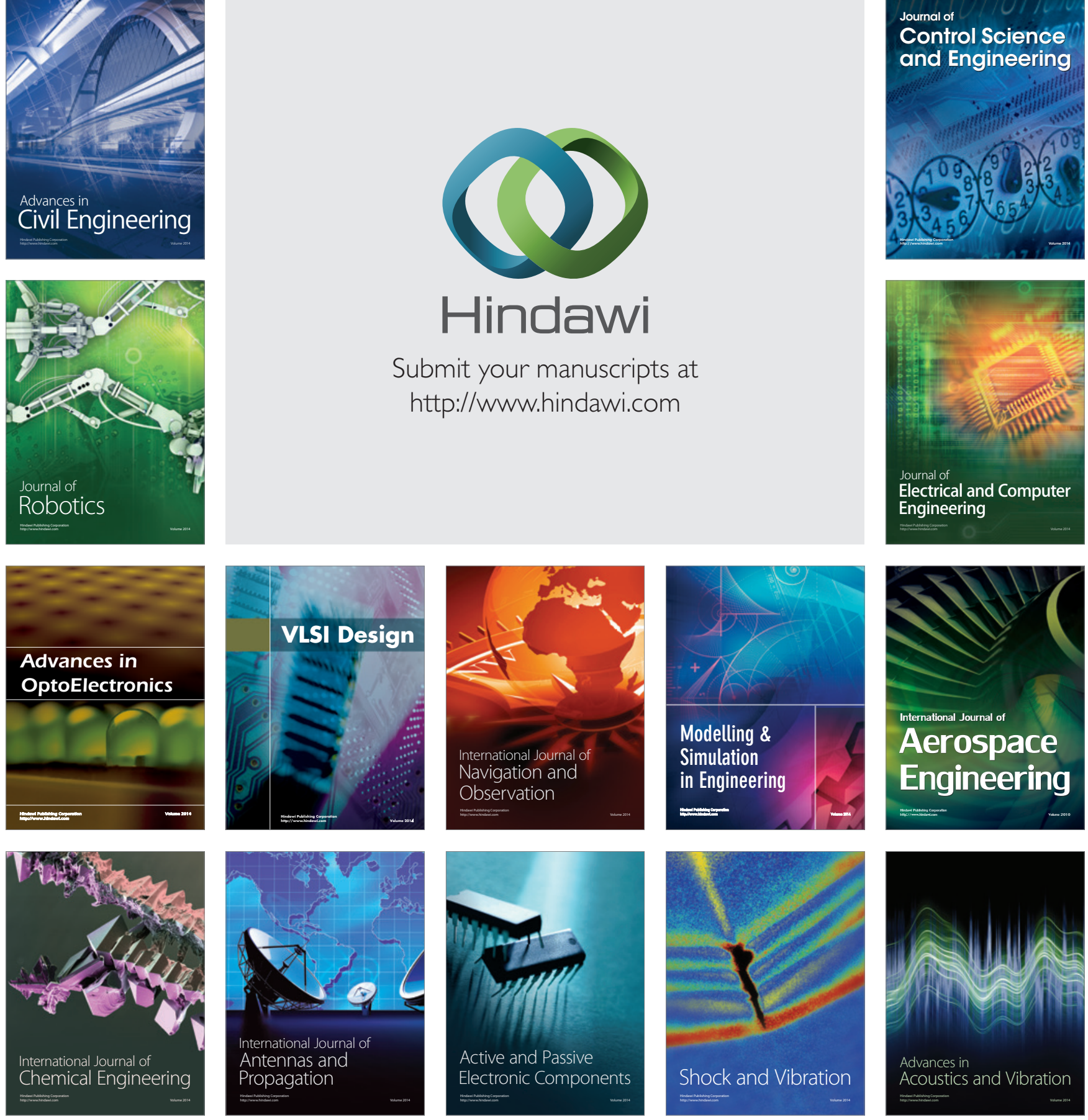